\title{
A Fuzzy Preprocessing Module for Optimizing the Access Network Selection in Wireless Networks
}

\author{
Faisal Kaleem, ${ }^{1}$ Abolfazl Mehbodniya, ${ }^{2}$ Kang K. Yen, ${ }^{1}$ and Fumiyuki Adachi ${ }^{2}$ \\ ${ }^{1}$ Department of Electrical and Computer Engineering, Florida International University, Miami, FL 33174, USA \\ ${ }^{2}$ Department of Electrical and Communication Engineering, Graduate School of Engineering, Tohoku University, \\ 6-6-05 Aza-Aoba, Aramaki, Sendai 980-8579, Japan \\ Correspondence should be addressed to Abolfazl Mehbodniya; mehbod@mobile.ecei.tohoku.ac.jp
}

Received 9 May 2012; Accepted 9 January 2013

Academic Editor: Harpreet Singh

Copyright (C) 2013 Faisal Kaleem et al. This is an open access article distributed under the Creative Commons Attribution License, which permits unrestricted use, distribution, and reproduction in any medium, provided the original work is properly cited.

\begin{abstract}
A heterogeneous wireless network is characterized by the presence of different wireless access technologies that coexist in an overlay fashion. These wireless access technologies usually differ in terms of their operating parameters. On the other hand, Mobile Stations (MSs) in a heterogeneous wireless network are equipped with multiple interfaces to access different types of services from these wireless access technologies. The ultimate goal of these heterogeneous wireless networks is to provide global connectivity with efficient ubiquitous computing to these MSs based on the Always Best Connected (ABC) principle. This is where the need for intelligent and efficient Vertical Handoffs (VHOs) between wireless technologies in a heterogeneous environment becomes apparent. This paper presents the design and implementation of a fuzzy multicriteria based Vertical Handoff Necessity Estimation (VHONE) scheme that determines the proper time for $\mathrm{VHO}$, while considering the continuity and quality of the currently utilized service, and the end-users' satisfaction.
\end{abstract}

\section{Introduction}

During recent years, the need for global connectivity to access different types of services at any place and any time has significantly increased. The latest innovations [1] in wireless access technology provide uninterrupted ondemand services, such as real-time multimedia, which are independent of device type, locations, and available networks [2]; the ultimate goal is to maintain a satisfactory end-user experience by providing the necessary quality and continuity of the currently utilized service in a cost-efficient manner. A heterogeneous wireless network comprises of different wireless networks including IEEE 802.15 based Wireless Personal Area Network (WPAN), IEEE 802.11 based Wireless Local Area Networks (WLAN), IEEE 802.16 based Wireless Metropolitan Area Network (WMAN), different types of cellular technologies such as Global System of Mobile Communication (GSM) and Universal Mobile Telecommunication System (UMTS), Vehicular Ad hoc Network (VANET), and satellite networks. To provide seamless mobility and convergence, integration of these diverse wireless networks is required. A multimodal Mobile Station (MS), in the presence of these integrated networks with overlapping coverage, can connect to any of these wireless access technologies. An MS, in a heterogeneous wireless network, switches its current Point of Attachment (PoA) to a new wireless network using a process called Vertical Handoff (VHO). These VHOs are required to maintain the continuity and quality of the current session while the MS moves between different wireless access technologies. Traditional handoff schemes are based on a single metric such as Received Signal Strength (RSS) and do not take into account factors such as the Quality of Service (QoS), end-user preferences, MS mobility and its location, and application contexts. In addition, the MS in a heterogeneous wireless environment has the capability to establish and maintain connectivity with many overlay networks that offer varying QoSs. Hence, estimating the necessity of a vertical handoff and choosing the right initiation time reduce the unnecessary handoffs, improves the overall QoS, and limits the inherent data signaling and rerouting in a VHO process, resulting in a maximized end-user's satisfaction. 
Handoff initiation techniques are studied in [3-10]. The scheme in [3] utilizes fuzzy logic with Multiple Objective Decision Making (MODM) approach to select the best network segment. The associated weights of the network parameters are obtained using the Analytic Hierarchy Process (AHP). This scheme does not take advantage of a full Fuzzy Inference System (FIS) and only utilizes the fuzzifier to calculate the membership values of the network parameters. Furthermore, only perceived QoS, without using the QoS-related parameters, is considered. The authors in [4] implement handoff initiation scheme by combining multiple parameters of all available networks in a cost function. All the available networks including the current PoA are then ranked using the AHP algorithm. However, the selection of the target network is done using an FIS that only utilizes two input parameters such as velocity and available bandwidth. The lack of other important parameters might not produce optimal results. Furthermore, not all the QoS parameters are taken into consideration while calculating the handoff initiations. A QoS-aware fuzzy logic based multicriteria algorithm is proposed in [5]. AHP is utilized to calculate the priority weights of network parameters. Only QoS-related parameters are considered to create four fuzzy logic controllers (FLCs) for four different traffic types. The major issue with this scheme is that it relies on bulky rule sets ( 81 rules), which makes it inefficient. Furthermore, the absence of RSS, MS speed, and other important parameters may result in nonoptimal handoff decisions. The authors in [6] utilize fuzzy logic with RSS and QoS to estimate the necessity of handoff. However, it is not shown how QoS is calculated and if all the necessary parameters are utilized to calculate it based on the requirements of different traffic classes. Furthermore, the MS's speed and its direction of movement are not considered; these factors play an important role in estimating the need of handoffs. The Artificial Intelligence (AI) scheme in [7], which is based on a hybrid of parallel fuzzy logic system, multiplecriteria decision making, and Genetic Algorithm (GA), is developed to provide adaptive, flexible, and scalable solution to the VHO decision problem. The decision phase uses three parallel fuzzy logic subsystems. The normalized outputs of these subsystems along with their importance weights, optimized using GAs, are fed into a multicriteria decision making system. This system is based on an enhanced version of Simple Multiattribute Rate Technique (SMART). The results show an increase percentage of satisfied users. However, the proposed scheme is limited to only four different criteria and does not take into consideration other important decision factor like loading conditions of the network. Furthermore, single-objective GAs are used to optimize each objective weight independently rather than utilizing a multiobjective utilization method to find optimal weights jointly, which could have resulted in an improved performance. A fuzzy multicriteria $\mathrm{VHO}$ algorithm with its parameters enhanced by the use of an inverted 2-layer Multilayer Perceptron (MLP) is proposed in [8]. In the proposed approach, a preliminary selection of candidate networks is performed using RSS to reduce the complexity of FLC. The FLC takes five inputs including RSS and loading conditions of the current and the target systems, and the velocity of MS. A total of 24 fuzzy handoff rules including general rules, UMTS specific rules, and the WLAN specific rules are created. In the proposed approach a 2-layer MLP, with FLC parameters as inputs and the desired UMTS and WLAN throughput as outputs, is trained and then inverted using a nonlinear system. This approach is compared against an algorithm that is based on fixed coverage and load thresholds. However, wireless networks are highly dynamic in nature resulting in varying load conditions and coverage. The authors in [9] utilize AHP for both weight elicitation and network selection processes. RSS is the only criterion that is used to trigger the handoff. This work is extended in [10] where authors implemented AHP based weight elicitation process along with Techniques for Order Preference by Similarity to Ideal Solution (TOPSIS) to rank the available networks. However, not all the QoS parameters are utilized to make selection decisions.

In this paper, we propose a module to estimate the necessity of vertical handoffs in a hybrid wireless environment. The proposed scheme utilizes fuzzy logic and AHP to determine if a $\mathrm{VHO}$ is required based on the measured values of the network parameters obtained from the serving PoA and other available wireless networks. A VHO factor is calculated using multiple parameters, including predicted value of RSS, the degree of provided QoS, the speed of the MS including its moving direction (towards/away from the serving $\mathrm{PoA}$ ), and the distance between the MS and the serving PoA. To determine the need for handoff, this calculated VHO factor is then compared against a threshold value that is predetermined based on the type (WLAN, WMAN, or WWAN) of the serving PoA. Grey Prediction Theory (GPT) is utilized to predict the future values of RSS that can minimize the call dropping probability of the MS due to a sudden drop in RSS, which is common in lognormal fading heterogeneous wireless networks. The degree of QoS is calculated based on the requirements of currently utilized traffic class type (conversational, interactive, background, or streaming) using all the necessary parameters such as throughput, jitter, delay, and Packet Loss Rate (PLR). To satisfy these requirements, our scheme calculates and assigns proper weights for a respective traffic class, using different techniques such as AHP, a fuzzy version of AHP (FAHP), and a fuzzy scheme based on the usage of linguistic variables and Triangular Fuzzy Numbers (TFNs). Several parallel FLCs with reduced rule sets are implemented to normalize the network parameters (measured from in-range wireless access technologies) as well as to calculate the vertical handoff factor. The proposed handoff necessity estimation scheme is the first module of an overall handoff decision algorithm. In case the handoff is deemed necessary, the second module in the handoff algorithm performs the selection of a target PoA; the selection is based on ranking algorithms that are designed to prioritize the potential target networks for VHO. Three Multiattribute Decision Making (MADM) based methods were utilized for developing the second module, being TOPSIS with AHP weighting, TOPSIS with FAHP weighting, and Fuzzy TOPSIS (FTOPSIS) with linguistic variables [11]. Our scheme is evaluated by providing numerical examples and by using a comprehensive simulation test bed that simulates a practical heterogeneous wireless environment with three 


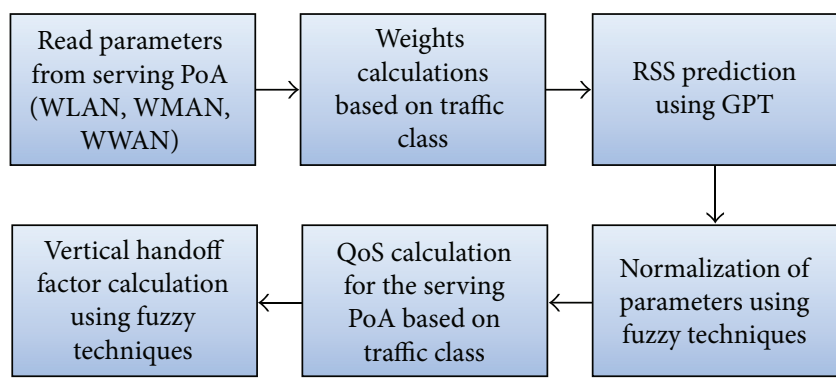

FIGURE 1: VHONE block diagram.

coexisting networks such as WLAN, WMAN, and WWAN. Different Radio Resource Management (RRM) subsystems including channel allocation, call admission, mobility, propagation, and traffic are implemented to compare our proposed scheme against a reference algorithm, that is, RSS-based algorithm with load balancing. Performance metrics such as average outage probability and handoff rate are used to evaluate our scheme.

The remainder of this paper is organized as follows. In Section 2, the proposed scheme is discussed. Section 3 outlines the simulation environment. In Section 4 , simulation results are provided using different performance metrics. Finally, in Section 5, concluding remarks are presented.

\section{Proposed Scheme}

A wireless environment is characterized by its dynamic nature, inherent uncertainty, and imprecise parameters and constraints. Network parameters like throughput, RSS, network delays, and so forth are intrinsically imprecise. Due to this vagueness, the accurate measurement of these network parameters in a wireless environment is a difficult task. As a result, a fuzzy logic approach seems to yield better results when used for system design in such environments. Although, Fuzzy Logic might be slower for vertical handoff algorithms and classical crisp value based techniques can be used to reduce handoff latency, these classical techniques are unable to produce intelligent and efficient handoff decisions. Hence, our scheme incorporates fuzzy logic based techniques to perform handoff necessity estimation and target selection.

The block diagram of the proposed VHONE module is depicted in Figure 1. The proposed module is capable of performing handoffs in case of a degraded QoS and weak RSS, and if certain other conditions, such as unavailability of a channel, become true. In the following, details of each component providing support to the VHONE module are provided.

2.1. System Parameters. The proposed scheme utilizes parameters such as RSS, MS speed and the direction (towards/away from current PoA) in which it is moving, distance between the current PoA and the MS, and the QoS of the serving network. These parameters are monitored and evaluated by VHONE module to determine if VHO is imminent. Although, scheme like [4] considers the MS battery status, the
TABLE 1: Operating ranges of network parameters.

\begin{tabular}{lccc}
\hline & WLAN & WMAN & WWAN \\
\hline RSS $(\mathrm{dBm})$ & $(-110)-(-55)$ & $(-160)-(-100)$ & $(-150)-(-90)$ \\
Delay $(\mathrm{ms})$ & $100-150$ & $10-50$ & $10-75$ \\
Jitter $(\mathrm{ms})$ & $10-30$ & $3-12$ & $5-15$ \\
PLR per $10^{6}$ bytes $(\%)$ & $3-7$ & $1-8$ & $1-5$ \\
Throughput (Mbps) & $50-150$ & $20-100$ & $0.1-3$ \\
Network range (m) & $0-100$ & $0-350$ & $0-750$ \\
Velocity (mps) & & $0-10$ & \\
\hline
\end{tabular}

proposed scheme ignores it as the end-user can control this parameter by connecting a battery charger while travelling. It is assumed that these parameters are available to the MS through some mechanism; for example, Global Positioning System (GPS) module installed in most modern MSs is capable of estimating the MS speed and direction. For simplicity, we also assume that the MS is capable of connecting to different types of wireless networks such as WLAN, WMAN, and WWAN, but at a given instant of time it is connected to only one specific type. The operating ranges of the network parameters that are considered by our scheme are shown in Table 1.

2.2. Weight Calculations for System Parameters. In order to specify the needs and preferences of end-user and to differentiate between the different traffic classes, priority weights are calculated and assigned for each of these network parameters. Since the goal of VHONE is to maximize end user's satisfaction in terms of quality and continuity of the received service, higher weights are assigned to RSS and QoSrelated parameters. Furthermore, since traffic types such as conversational, streaming, interactive, and background have varying QoS requirements [12], weights for different QoSrelated parameters including throughput, jitter, delay, and PLR are also calculated and assigned. Note that these weights can be assigned manually or calculated following certain weight elicitation techniques. For more details, readers may refer to [13].

2.3. RSS Prediction Using Grey Prediction Theory. In a lognormal fading wireless environment, higher rate of handoffs as well as unnecessary handoffs may affect the quality of the provided service, resulting in reduced end-users' satisfaction. Although, RSS with threshold and hysteresis approaches [14] can minimize the number of unnecessary handoffs, these schemes result in a low data rate and high call dropping probabilities since, at the time of handoffs, the RSS reception from the current PoA may become too weak. In [15] a scheme based on GPT [16] is proposed to perform horizontal handoffs; we use GPT to determine if a future vertical handoff is required based on the predicted value of RSS measured from the current PoA. Table 2 shows Predicted RSS (PRSS) values using GPT for three network types. While a continuous drop pattern for RSS can be observed for both WLAN and WMAN networks, predicted value of a weaker RSS, calculated using GPT, can help reduce the unnecessary 
TABLE 2: PRSS using GPT.

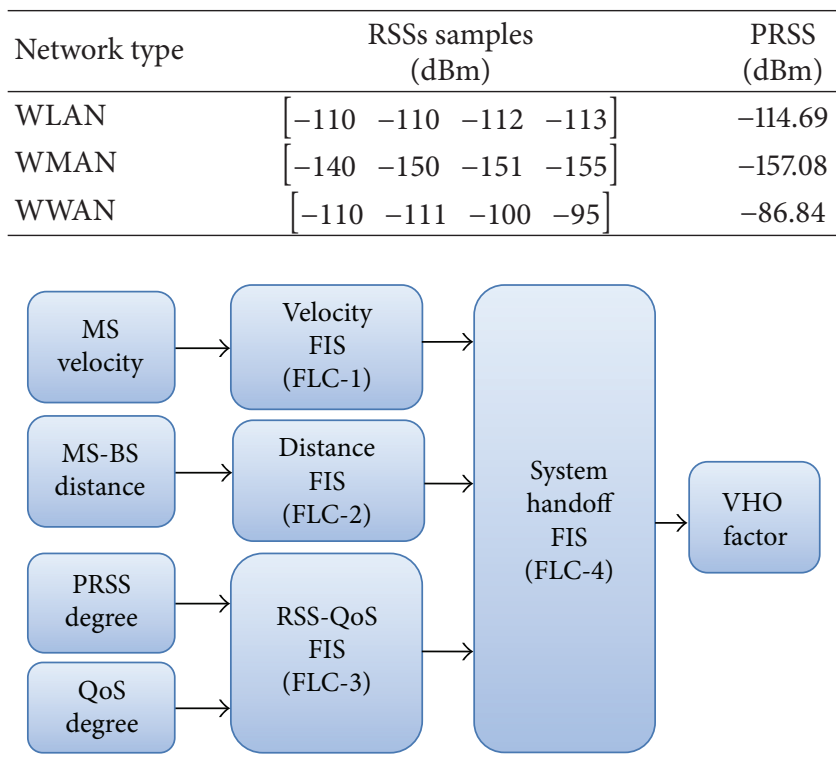

FIGURE 2: VHO factor calculations.

call drops. Readers may refer to [13] for mathematical details regarding GPT based RSS prediction.

2.4. Normalization of Network Parameters. The operating parameters measured from different types of wireless networks in a heterogeneous environment are generally dissimilar and are not directly comparable. For example, the RSS ranges of WLAN and WMAN are quite different. Therefore, a high value of RSS measured from a WLAN may not be considered high in a WMAN environment. This research work proposes the use of parallel FLCs (Figure 2) to normalize all parameters by calculating their respective membership values in the range of $(0,1)$. Fuzzy sets for each parameter, with their respective Universe of Discourses (UoDs) based on published standards [17, 18], are created.

Different linguistic variables such as Low, Medium, and High are used to partition these UoDs. Due to their computational simplicity, trapezoidal membership functions are used to represent these fuzzy sets according to

$$
\mu_{A}(x)= \begin{cases}0 & x<l ; x>u, \\ \frac{x-l}{c-(w / 2)-l}, & l<x<\left(c-\frac{w}{2}\right), \\ \frac{u-x}{u-(w / 2)-c}, & \left(c+\frac{w}{2}\right)<x<u, \\ 1, & \left(c-\frac{w}{2}\right)<x<\left(c+\frac{w}{2}\right),\end{cases}
$$

where $l$ and $u$ are the lower and upper bounds, respectively. $c$ is the center and $w$ is the width of the top side of the symmetric trapezoid. Since the network parameters can be classified as benefit type or cost type, their normalization is performed using specific fuzzy inference rules that maximize the membership values of benefit types and minimize the
TABLE 3: Rule set for WMAN-RSS.

\begin{tabular}{|c|c|}
\hline $\begin{array}{l}\text { Rule } \\
\text { number }\end{array}$ & Rule description \\
\hline 1 & If WMAN-RSS is Low then WMAN-Select is Low \\
\hline 2 & $\begin{array}{l}\text { If WMAN-RSS is Medium then WMAN-Select is } \\
\text { Medium }\end{array}$ \\
\hline 3 & If WMAN-RSS is High then WMAN-Select is High \\
\hline & TABLE 4: Rule set for WMAN-lantecy. \\
\hline $\begin{array}{l}\text { Rule } \\
\text { number }\end{array}$ & Rule description \\
\hline 1 & If WMAN-Latency is Low then WMAN-Reject is Low \\
\hline 2 & $\begin{array}{l}\text { If WMAN-Latency is Medium then WMAN-Latency is } \\
\text { Medium }\end{array}$ \\
\hline 3 & $\begin{array}{l}\text { If WMAN-Latency is High then WMAN-Latency is } \\
\text { High }\end{array}$ \\
\hline
\end{tabular}

values of cost type parameters, respectively. For benefit type parameter such as RSS, FLC produces a probability for MS selecting the network based on measured RSS. Similarly, for cost type parameters such as latency, the respective FLC estimates a probability for MS rejecting the network based on the latency value measured from that network. Tables 3 and 4 , respectively, show the rule sets for FISs that are developed for RSS (a benefit type parameter) and Latency (a cost type), both measured from WMAN. Figure 3 shows the Fuzzy set representing RSS for WMAN, whereas Figure 4 depicts the FLC for RSS in a WAMN environment, which is used for normalizing the values of RSS. Based on the rules in FIS, the FLC produces a singleton value in the range of $(0.0,1.0)$ that acts as a normalized value for that parameter. In a similar fashion, the values of other parameters are normalized as well.

2.5. Calculation of Degree of QoS for Current PoA. RSS and QoS are two important factors that play a critical role in determining the end-user's satisfaction. Hence, based on the current service type, the proposed scheme monitors the QoS-related parameters provided by the serving PoA and calculates the degree of QoS in the range of $(0,1)$ according to

$$
\begin{aligned}
\text { QoS }_{\text {degree }}= & {\left[\mu^{\text {Delay }} \mu^{\text {Jitter }} \mu^{\text {PLR }} \mu^{\text {Throughput }}\right] } \\
& \times\left[W_{\mathrm{TC}}^{D} W_{\mathrm{TC}}^{J} W_{\mathrm{TC}}^{P} W_{\mathrm{TC}}^{T}\right]^{T},
\end{aligned}
$$

where $W_{\mathrm{TC}}^{X}$ is the weight of the QoS parameter $X$ (delay, jitter, PLR, and throughput) for a particular traffic class TC (conversational, streaming, background, or interactive). Equation (2) calculates a weighted sum of membership values of QoS parameters (delay, jitter, PLR, and throughput). Any variations in these parameters affect the overall degree of QoS. Furthermore, since QoS requirements are different for various types of traffic classes, different weights with respect to these traffic types need to be calculated and assigned, specifically for QoS-related parameters. These weights are calculated using different MADM algorithms utilizing 


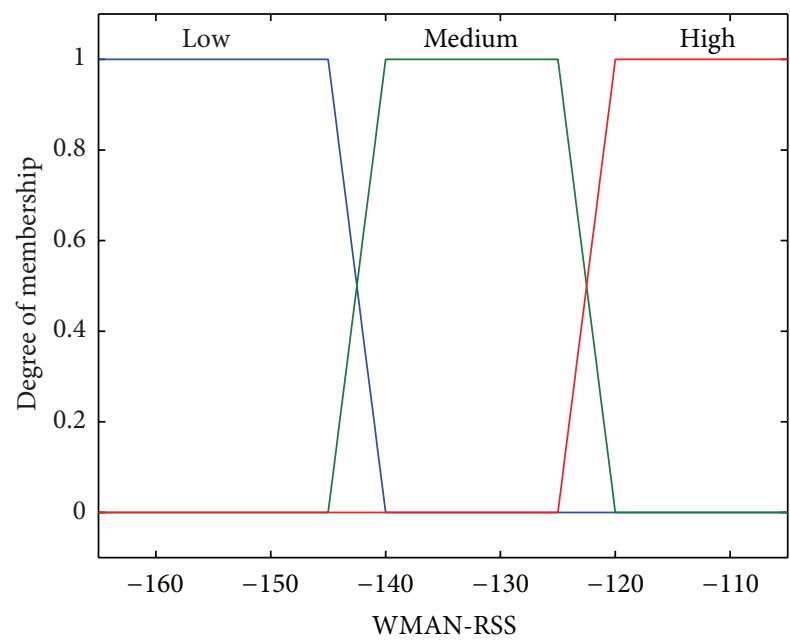

FIgURE 3: Fuzzy set representing RSS (WMAN).

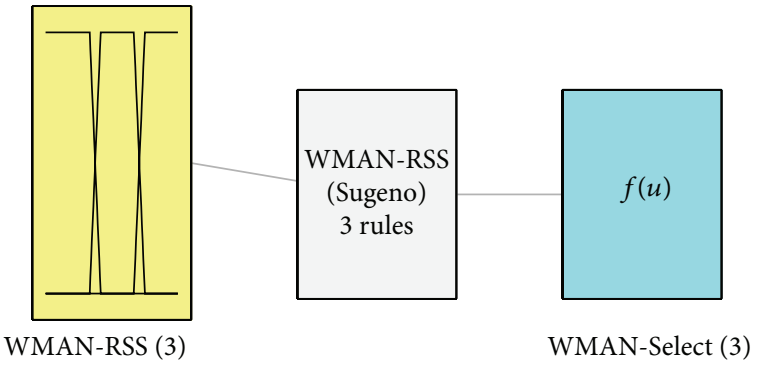

FIGURE 4: FLC for RSS (WMAN).

the different characteristics and QoS demands for the four types of traffic classes as defined by 3GPP TS-23.107 specification [12].

2.6. VHO Factor Calculation Using Fuzzy Logic. Based on the current conditions of serving PoA, four different FLCs are utilized to calculate the value of VHO factor that in turn determines the necessity of handoffs. In order to reduce the number of rules and system complexity, three fuzzy logic controllers are combined in a parallel fashion. The outputs of these three FLCs are then fed into the fourth fuzzy logic controller that produces the final VHO factor. With carefully designed rules, both Sugeno [19] and Mamdani [20] type FISs are incorporated. This is shown in Figure 2. In the following paragraphs, the details of these four fuzzy logic controllers are provided. Velocity is considered an important factor in the proposed scheme. To reduce the outage probability and the unnecessary handoffs, MSs with higher speeds are connected to networks with larger coverage areas such as WWAN. A Sugeno based FLC with one input (MS-Velocity) and three output variables are utilized. The input variable has three membership functions (Low, Medium, and High) and each of the three output variables has three singleton membership functions (Low, Medium, and High). The output variables reflect a probability of rejection for an MS to be in a specific network type at a given input speed. Table 5
TABLE 5: Inference rule for FLC-1.

\begin{tabular}{ll}
\hline $\begin{array}{l}\text { Rule } \\
\text { number }\end{array}$ & Rule description \\
\hline 1 & $\begin{array}{l}\text { If MS Velocity is Low then WLAN-Reject is Low, } \\
\text { WMAN-Reject is Medium, and WWAN-Reject is High }\end{array}$ \\
2 & $\begin{array}{l}\text { If MS Velocity is Medium then WLAN-Reject is High, } \\
\text { WMAN-Reject is Low, and WWAN-Reject is Medium }\end{array}$ \\
3 & $\begin{array}{l}\text { If MS-Velocity is High then WLAN-Reject is High, } \\
\text { WMAN-Reject is Medium, and WWAN-Reject is Low }\end{array}$ \\
\hline
\end{tabular}

shows the inference rules for FLC-1. Distance between the MS and the current PoA (BS/AP) also plays a critical role in determining handoffs. As the distance between the MS and the current PoA increases, the measured values of RSS and other critical factors decrease. Thus, handoff becomes imminent. The FLC-2 in the proposed scheme is designed based on the coverage provided by a specific network type. Since the coverage areas of the three types of networks are different and the assumption that at the most MS will be connected to one PoA, separate membership functions with different UoDs are designed based on these network types. A Sugeno based FLC with one input (distance between MS and AP) and one output (probability of rejection for a network type) is created for each network type.

Since the main objective of the proposed scheme is to maximize the end-user's satisfaction in terms of the quality and continuity of the currently utilized service, RSS and QoS play a very important role to achieve this objective. If any of these two parameters fall below a minimum, the overall quality of the current application session diminishes resulting in reduced end-user's satisfaction. The FLC-3 is designed to make sure that the MS performs handoff before any of these two factors fall below the minimum network values required to sustain the quality of the currently utilized service. Since the QoS measurements vary from one network type to another, separate FLCs are designed for each of the three network types. A Mamdani based FLC with two inputs (Network PRSS degree and Network QoS degree) and one output is utilized. As an example, the inference rules of FLC3 for WLAN are shown in Table 6. These rules indicate that a low value of both inputs results in a high value for the output variable, PRSS-QoS Factor, indicating a higher probability of handoff. Similar FLCs are designed for WMAN and WWAN. The only difference between these three FLCs is the incorporation of different UoDs with different ranges for each network types due to the varying QoS values.

The FLC-4 is the main controller that determines the necessity of VHOs based on the existing conditions of serving PoA. These conditions are evaluated using parallel FLCs to generate three different factors. These factors serve as inputs to Mamdani based FLC-4, which in turn outputs the VHO factor for the current PoA. Note that the PoA can be WLAN, WMAN, or WWAN. A reduced number of rules (19 instead of 27) are implemented due to the fact that the proposed scheme gives more importance to the RSS-QoS Factor as it plays a critical role in determining the quality of the currently utilized service and maximizing the end-user's satisfaction. 
TABLE 6: Inference rules for FLC-3.

\begin{tabular}{|c|c|}
\hline Rule number & Rule description \\
\hline 1 & $\begin{array}{l}\text { If PRSS-deg is High and QoS-deg is Low then } \\
\text { P-Q-Factor is High }\end{array}$ \\
\hline 2 & $\begin{array}{l}\text { If PRSS-deg is High and QoS-deg is Medium } \\
\text { then P-Q-Factor is Medium }\end{array}$ \\
\hline 3 & $\begin{array}{l}\text { If PRSS-deg is High and QoS-deg is High then } \\
\text { P-Q-Factor is Low }\end{array}$ \\
\hline 4 & $\begin{array}{l}\text { If PRSS-deg is Medium and QoS-deg is Low } \\
\text { then P-Q-Factor is High }\end{array}$ \\
\hline 5 & $\begin{array}{l}\text { If PRSS-deg is Medium and QoS-deg is } \\
\text { Medium then P-Q-Factor is Medium }\end{array}$ \\
\hline 6 & $\begin{array}{l}\text { If PRSS-deg is Medium and QoS-deg is High } \\
\text { then P-Q-Factor is Medium }\end{array}$ \\
\hline 7 & $\begin{array}{l}\text { If PRSS-deg is Low and QoS-deg is Low then } \\
\text { P-Q-Factor is High }\end{array}$ \\
\hline 8 & $\begin{array}{l}\text { If PRSS-deg is Low and QoS-deg is Medium } \\
\text { then P-Q-Factor is High }\end{array}$ \\
\hline 9 & $\begin{array}{l}\text { If PRSS-deg is Low and QoS-deg is High then } \\
\text { P-Q-Factor is High }\end{array}$ \\
\hline
\end{tabular}

The details for each membership functions of each parameter are omitted due to space limitation. However, readers may refer to [13] for more information.

2.7. Handoff Necessity Estimation. The final handoff factor that is obtained as the output from FLC- 4 is compared against a predetermined threshold value to determine if a handoff from the serving PoA is required. This threshold value, in the range of $(0.0,1.0)$, is calculated and optimized after performing numerous simulation rounds and observing the $\mathrm{VHO}$ factor that is calculated by the VHONE module. Note that a higher value of this threshold will prevent necessary handoffs, resulting in high probability of call drops. On the other hand, a low value will result in frequent and costly handoffs, resulting in unnecessary wastage of system resources. Thus a balanced value for this threshold is required. This balanced value is achieved by performing numerous simulations and observing the need of handoffs based on different input conditions. Due to the technology difference, this value can be chosen differently for different types of networks, can be varied for different simulation setups and numerical values of the parameters, and can be adjusted according to the sensitivity of the network types. However, for the sake of simplicity, the proposed scheme utilizes a value of 0.75 for all three network types.

\section{Simulation Environment}

The VHONE is implemented using MATLAB and evaluated using a comprehensive test bed developed based on the concept of RUNE [21]. RUNE is a special purpose simulator to simulate wireless networks. Several RRM modules including mobility, propagation, and traffic are created employing a cellular concept for three coexisting networks, that is, WLANs, WMANs, and WWANs.
The WLAN is defined with 27 cells with a radius of 100 meters each. The WMAN and WWAN are defined with 12 cells, each with a radius of 375 and 750 meters, respectively. For all three network types, the standard hexagonal shape with omnidirectional antennas is considered for each cell within a network. A cluster of 3 cells is formed and the total frequency range for each network is divided among these 3 cells. These divided frequencies are repeated at each cluster. This arrangement is kept the same for all three network types. The total number of available channels per cell is kept as 8, 12 , and 16 for WLAN, WMAN, and WWAN, respectively. Channels of different networks are assumed to be orthogonal. We consider path loss, shadow fading, and Rayleigh fading in the propagation model.

For the performance evaluation, we consider a multiuser scenario where several MSs join the system based on a Poisson arrival rate and the connection duration is modeled based on an exponential distribution. A mobility model similar to [21] is considered where new MSs are distributed uniformly in the environment and the new direction and velocity of each MS is updated randomly based on a specific correlation with the previous values. Simulations are done utilizing complete VHO algorithm with two modules: VHONE as the first module and a second module for ranking the target networks based on TOPSIS-AHP, TOPSIS-FAHP, and FTOPSIS algorithms. Two metrics are considered to evaluate the proposed scheme, that is, average system outage probability and the handoff rate. We compare the performance of our proposed scheme with an existing $\mathrm{VHO}$ algorithm that combines the RSS threshold comparison and network load balancing. Evaluations are done based on different number of arrived calls per cell and multiple MSs moving randomly at the speeds of $1 \mathrm{~m} / \mathrm{s}, 5 \mathrm{~m} / \mathrm{s}$, and $9 \mathrm{~m} / \mathrm{s}$. Conversational traffic class is utilized for obtaining the results.

\section{Performance Evaluation}

Table 7 depicts parameter value sets for two chosen scenarios. Please note that besides typical parameter values, the fuzzy variables like Low, Medium, High, Near, and Far are also introduced in this table to provide a better understanding for the readers. Set- 1 shows the different settings and parameter values utilized assuming that the end-user is currently watching a recorded webcast (streaming) using his/her home's WLAN. The GPT predicted higher value of RSS combined with the stationary $(0 \mathrm{~m} / \mathrm{s}) \mathrm{MS}$, located at a short distance from WLAN based Access Point (AP), results in a low value of 0.25 . This value indicates an overall handoff factor that the VHONE module calculated based on values for parameter set-1. Since this value is less than the handoff threshold, currently set at 0.75 for WLAN, the MS does not perform the handoff and remains connected to its current PoA (WLAN).

The second scenario is based on the assumption that the end-user leaves home for work and starts walking towards the nearest bus stand while watching the same webcast. The parameter values in set-2 (depicted in Table 7) are utilized for this scenario. As the user walks away from his/her home, 
TABle 7: Parameter sets for VHONE.

\begin{tabular}{lcc}
\hline Parameters & $\begin{array}{c}\text { Values } \\
(\text { Set-1) }\end{array}$ & $\begin{array}{c}\text { Values } \\
\text { (Set-2) }\end{array}$ \\
\hline Current PoA & WLAN & WLAN \\
Current traffic class & Streaming & Streaming \\
Metric weighting algorithm scheme & AHP & AHP \\
MS velocity $(\mathrm{m} / \mathrm{s})$ & 0 (Low) & 1 (Low) \\
MS-PoA distance $(\mathrm{m})$ & 10 (Near) & 85 (Far) \\
RSS samples $(\mathrm{dBm})$ & $-58.5,-55.3$, & $-90.5,-92.7$ \\
& $-57.6,-59.8$ & $-97.3,-98.9$ \\
PRSS using GPT $(\mathrm{dbm})$ & -62.21 & -102.63 \\
& $($ High $)$ & $($ unreachable) \\
Delay (ms) & 100 (Low) & 120 (High) \\
Jitter (ms) & 10 (Low) & 20 (High) \\
PLR (loss per $10^{6}$ bytes) & 3 (Low) & 4 (Medium) \\
Throughput $(\mathrm{Mbps})$ & 130 (High) & 30 (Low) \\
\hline
\end{tabular}

the distance between the WLAN-AP and MS increases and the RSS started to become weaker. Note that, based on the RSS samples, the GPT predicted an RSS value that cannot be sensed by the MS that is moving away from the WLAN-AP. Based on parameter set-2, the VHONE module calculates a VHO factor of 0.85 that is higher than the threshold. Hence, the module triggers the handoff in order to guarantee the continuity and the quality of the currently utilized service.

The following two metrics are considered to evaluate the proposed scheme. The average outage probability is a statistical measure that defines the probability of an MS failing to receive a signal with adequate quality at a particular location. This outage occurs when the SINR of an MS falls below a prescribed threshold. The necessity of handoff and the decision to select the best network as the target of handoff is related to this metric. A lower value of this metric indicates a high number of MSs that have received adequate signal strength, which in turn translate into intelligent and efficient network selection decisions made by the proposed scheme. The average handoff rate is defined as the number of handoffs that the MS has performed during a call connection. The metric is critically important as it affects the quality of the ongoing service. A lower value of this metric is desirable to maximize end-user satisfaction in terms of guaranteed continuity and quality of service. It is important to mention that the average handoff rate is directly proportional to the MS battery consumption. The higher the handoff rate, the more quickly the MS battery drains.

Figures 5, 6, and 7 show the average system outage probabilities offered by three different algorithms for call arrivals per cell (1-10) based on conversational traffic type. It can be observed that VHONE scheme utilizing TOPSIS-AHP, TOPSIS-FAHP, and FTOPSIS provides significant performance improvement over existing RSS with load-balancingbased scheme. For MSs moving with any speed, FTOPIS outperforms the other algorithms in terms of average outage probability; for example, with maximum number of calls per cell (10), an MS moving at a speed of $9 \mathrm{~m} / \mathrm{s}$

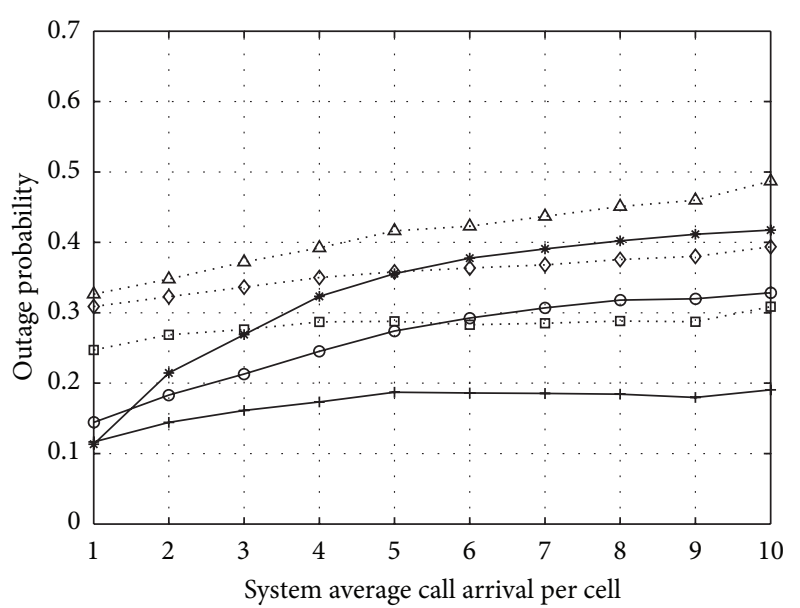

— TOPSIS-AHP, conversational traffic, Low velocity $(1 \mathrm{~m} / \mathrm{s})$

$\rightarrow$ TOPSIS-AHP, conversational traffic, Medium velocity $(5 \mathrm{~m} / \mathrm{s})$

* TOPSIS-AHP, conversational traffic, High velocity $(9 \mathrm{~m} / \mathrm{s})$

... RSS based, conversational traffic, Low velocity $(1 \mathrm{~m} / \mathrm{s})$

$\diamond \diamond$ RSS based, conversational traffic, Medium velocity $(5 \mathrm{~m} / \mathrm{s})$

$\Delta$ RSS based, conversational traffic, High velocity $(9 \mathrm{~m} / \mathrm{s})$

FIGURE 5: Outage probability using TOSPSIS-AHP.

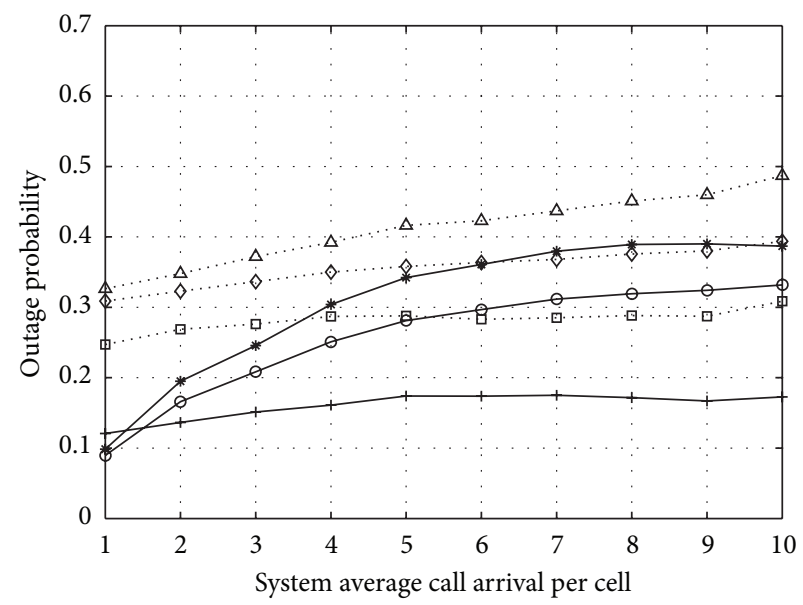

\footnotetext{
— TOPSIS-FAHP, conversational traffic, Low velocity $(1 \mathrm{~m} / \mathrm{s})$

$\rightarrow$ TOPSIS-FAHP, conversational traffic, Medium velocit $(5 \mathrm{~m} / \mathrm{s})$

- - TOPSIS-FAHP, conversational traffic, High velocity $(9 \mathrm{~m} / \mathrm{s})$

. •. RSS based, conversational traffic, Low velocity $(1 \mathrm{~m} / \mathrm{s})$

$\cdots \diamond$ RSS based, conversational traffic, Medium velocity $(5 \mathrm{~m} / \mathrm{s})$

$\cdot \Delta \cdot$ RSS based, conversational traffic, High velocity $(9 \mathrm{~m} / \mathrm{s})$
}

FIGURE 6: Outage probability using TOPSIS-FAHP.

shows improvement of $17 \%$ over the RSS-based scheme. Figures 8, 9, and 10 depict the average handoff rate for the same algorithms utilized by VHONE. Once again, VHONE utilizing FTOPSIS performs better than the other algorithms with a significant reduction of handoff rate to $31 \%$ as compared to RSS-based scheme that shows a very high handoff rate of $71 \%$. The aforementioned handoff rates are calculated for an average call arrival rate of 10 per cell and with MSs' 


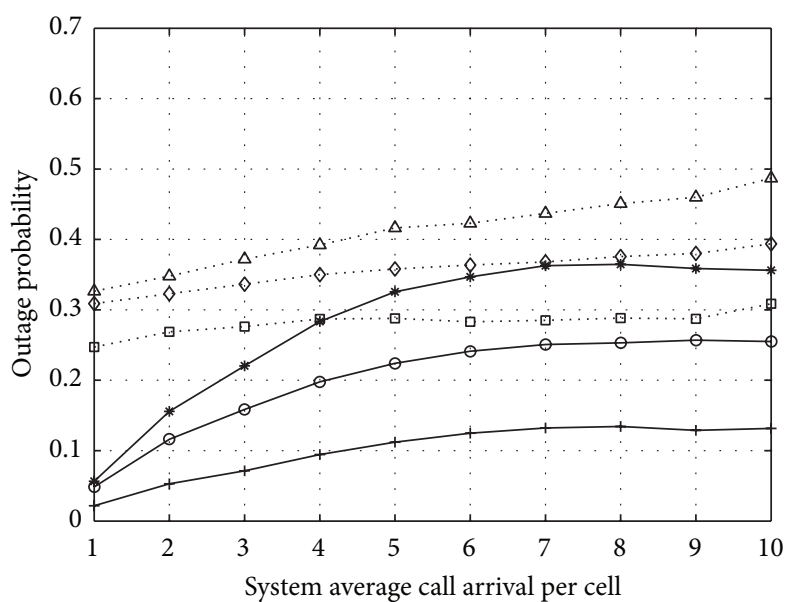

1- FTOPSIS, conversational traffic, Low velocity $(1 \mathrm{~m} / \mathrm{s})$

$\rightarrow$ FTOPSIS, conversational traffic, Medium velocit $(5 \mathrm{~m} / \mathrm{s})$

$\rightarrow$ FTOPSIS, conversational traffic, High velocity $(9 \mathrm{~m} / \mathrm{s})$

. .. RSS based, conversational traffic, Low velocity $(1 \mathrm{~m} / \mathrm{s})$

$\bullet \diamond$ RSS based, conversational traffic, Medium velocity $(5 \mathrm{~m} / \mathrm{s})$

$\Delta \cdot$ RSS based, conversational traffic, High velocity $(9 \mathrm{~m} / \mathrm{s})$

FIGURE 7: Outage probability using FTOPSIS.
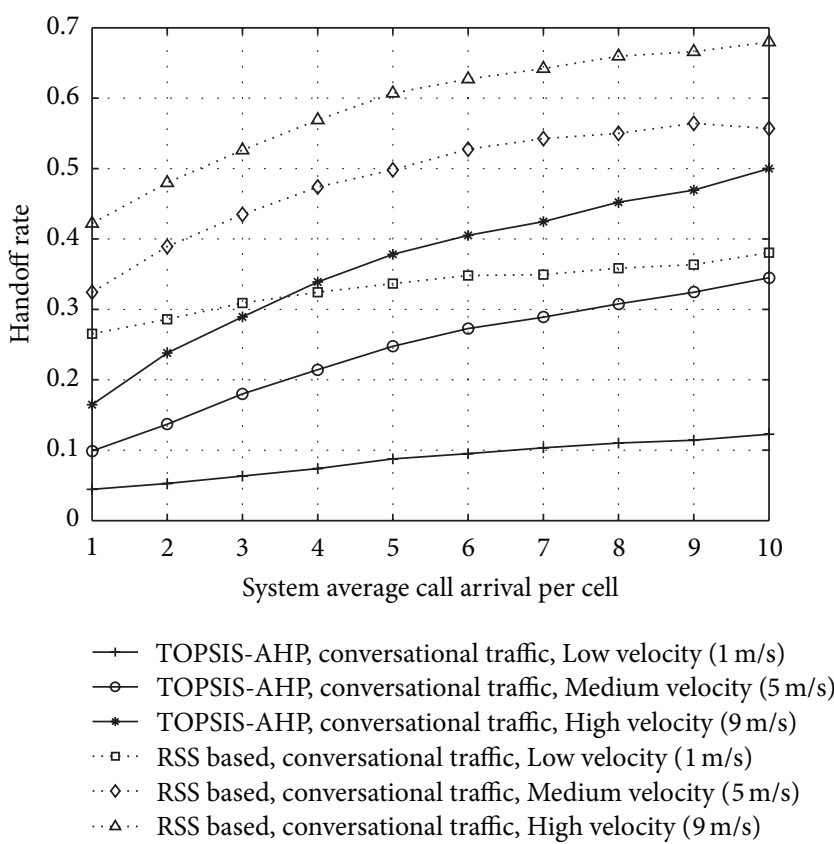

Figure 8: Handoff rate using TOPSIS-AHP.

speed of $9 \mathrm{~m} / \mathrm{s}$. These improvements over the existing RSSbased algorithm show that the proposed VHONE module is performing handoff necessity estimation in a more intelligent and efficient manner. This in turn reduces the ping-pong effect and conserves the limited battery life of the MS. Table 8 summarizes the percentages of outage probabilities and handoff rates for the conversational traffic class based on a maximum call arrival rate of 10 and for three different MS's speeds $(1,5$, and $9 \mathrm{~m} / \mathrm{s})$.

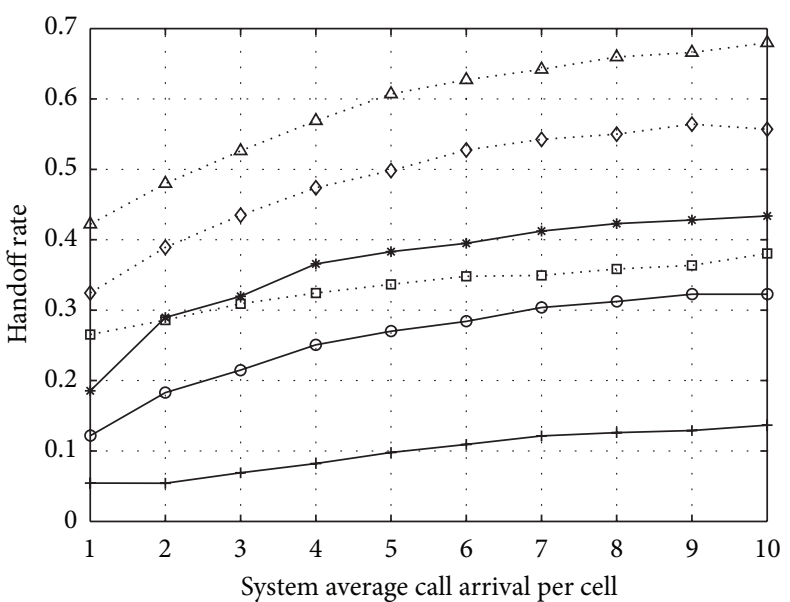

- TOPSIS-FAHP, conversational traffic, Low velocity $(1 \mathrm{~m} / \mathrm{s})$

$\rightarrow$ TOPSIS-FAHP, conversational traffic, Medium velocit $(5 \mathrm{~m} / \mathrm{s}$ )

* TOPSIS-FAHP, conversational traffic, High velocity $(9 \mathrm{~m} / \mathrm{s})$

. $\cdots$ RSS based, conversational traffic, Low velocity $(1 \mathrm{~m} / \mathrm{s})$

$\checkmark$. RSS based, conversational traffic, Medium velocity $(5 \mathrm{~m} / \mathrm{s})$

... RSS based, conversational traffic, High velocity $(9 \mathrm{~m} / \mathrm{s})$

Figure 9: Handoff rate using TOPSIS-FAHP.

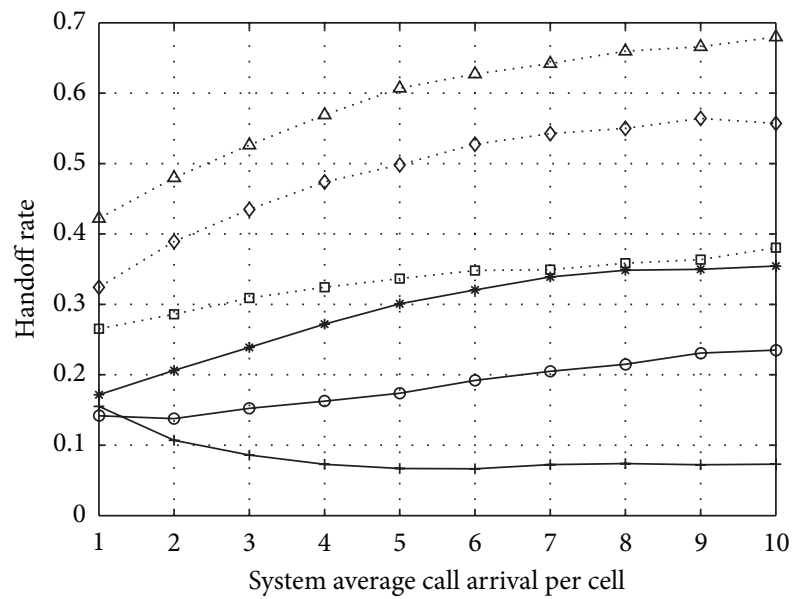

- FTOPSIS, conversational traffic, Low velocity $(1 \mathrm{~m} / \mathrm{s})$

$\rightarrow$ FTOPSIS, conversational traffic, Medium velocit $(5 \mathrm{~m} / \mathrm{s})$

$\rightarrow$ FTOPSIS, conversational traffic, High velocity $(9 \mathrm{~m} / \mathrm{s})$

$\therefore$ RSS based, conversational traffic, Low velocity $(1 \mathrm{~m} / \mathrm{s})$

$\because \diamond \cdot$ RSS based, conversational traffic, Medium velocity $(5 \mathrm{~m} / \mathrm{s})$

$\therefore \Delta$ RSS based, conversational traffic, High velocity $(9 \mathrm{~m} / \mathrm{s})$

FIgURE 10: Handoff rate using FTOPSIS.

\section{Conclusion}

A Vertical Handoff Necessity Estimation (VHONE) scheme was proposed to determine the proper time for a future handoff in order to preserve the continuity and the quality of the current session. Several parallel fuzzy logic controllers were utilized with multiple parameters measured from the current Point of Attachment (PoA) to calculate the necessity of vertical handoffs. This includes the predicted value of RSS 
TABLE 8: VHONE for conversational class.

\begin{tabular}{lcccccc}
\hline & \multicolumn{3}{c}{$\begin{array}{c}\text { Average outage } \\
\text { probability (\%) }\end{array}$} & \multicolumn{3}{c}{$\begin{array}{c}\text { Average handoff } \\
\text { rate (\%) }\end{array}$} \\
\hline Speed (m/s) & 1 & 5 & 9 & 1 & 5 & 9 \\
Call arrival & \multicolumn{3}{c}{$\begin{array}{c}\text { Max (10) } \\
\text { RSS }\end{array}$} & \multicolumn{4}{c}{ Max (10) } \\
TOPSIS-AHP & 17 & 31 & 52 & 39 & 60 & 71 \\
TOPSIS-FAHP & 16 & 31 & 40 & 16 & 33 & 43 \\
FTOPSIS & 14 & 28 & 35 & 11 & 22 & 31 \\
\hline
\end{tabular}

provide by the current PoA computed using Grey Prediction Theory (GPT), the degree of the provided QoS based on the current type of traffic class (conversational, streaming, interactive, or background), and the directional speed of the MSs. It was observed that our proposed scheme provided better results when compared against RSS load-balancingbased algorithm. For instance, using our FTOPSIS-based VHO algorithm, the overall system outage probability was improved by approximately $17 \%$ and handoff rate by $40 \%$, for MSs moving with a speed of $9 \mathrm{~m} / \mathrm{s}$ and at maximum system loading (10 calls per cell).

\section{References}

[1] I. F. Akyildiz, J. Xie, and S. Mohanty, "A survey of mobility management in next-generation all-IP-based wireless systems," IEEE Wireless Communications, vol. 11, no. 4, pp. 16-28, 2004.

[2] G. Lampropoulos, A. K. Salkintzis, and N. Passas, "Mediaindependent handover for seamless service provision in heterogeneous networks," IEEE Communications Magazine, vol. 46, no. 1, pp. 64-71, 2008.

[3] P. M. L. Chan, Y. F. Hu, and R. E. Sheriff, "Implementation of fuzzy multiple objective decision making algorithm in a heterogeneous mobile environment," in Proceedings of the Wireless Communications and Networking Conference ( WCNC '02), vol. 1, pp. 332-336, March 2002.

[4] Z. Yan, H. Luo, Y. Qin et al., "An adaptive multi-criteria vertical handover framework for heterogeneous networks," in Proceedings of the International Conference on Mobile Technology, Applications, and Systems, pp. 14:1-14:7, Yilan, Taiwan, September 2008.

[5] K. Vasu, S. Maheshwari, S. Mahapatra, and C. S. Kumar, "QoS aware fuzzy rule based vertical handoff decision algorithm for wireless heterogeneous networks," in Proceedings of the National Conference on Communications (NCC '11), pp. 1-5, January 2011.

[6] S. K. M. Venkata and L. Rajesh, "Implementation of fuzzy logic for network selection in next generation networks," in Proceedings of the International Conference on Recent Trends in Information Technology (ICRTIT '11), pp. 595-600, June 2011.

[7] M. Alkhawlani and A. Ayesh, "Access network selection based on fuzzy logic and genetic algorithms," Advances in Artificial Intelligence, vol. 2008, Article ID 793058, 12 pages, 2008.

[8] S. Horrich, S. Ben Jemaa, and P. Godlewski, "Neural networks for adaptive vertical handover decision," in Proceedings of the 5th International Symposium on Modeling and Optimization in Mobile, Ad Hoc, and Wireless Networks (WiOpt '07), pp. 1-7, April 2007.
[9] S. Dhar, A. Ray, and R. Bera, "Design and simulation of vertical handover algorithm for vehicular communication," International Journal of Engineering Science and Technology, vol. 2, pp. 5509-5525, 2010.

[10] S. Dhar, A. Ray, and R. Bera, "A context aware vertical handoff algorithm for vehicular communication," International Journal of Electronics, Computer and Communications Technologies, vol. 2, no. 1, pp. 24-34, 2011.

[11] F. Kaleem, A. Mehbodniya, K. K. Yen, and F. Adachi, "Application of fuzzy TOPSIS for weighting the system attributes in overlay networks," in Proceedings of the 14th Asia-Pacific Network Operations and Management Symposium (APNOMS '12), pp. 1-6, September 2012.

[12] J. Rinne, "3GPP Specification Details, TS 23. 107”.

[13] F. Kaleem, VHITS: vertical handoff initiation and target selection in a heterogeneous wireless network [Ph.D. dissertation], Florida International University, 2012.

[14] B. V. Quang, R. V. Prasad, and I. Niemegeers, "A survey on handoffs-lessons for $60 \mathrm{GHz}$ based wireless systems," IEEE Communications Surveys \& Tutorials, vol. 14, no. 1, pp. 64-86, 2010.

[15] C. H. Lee and C. J. Yu, "An intelligent handoff algorithm for wireless communication systems using grey prediction and fuzzy decision system," in Proceedings of IEEE International Conference on Networking, Sensing and Control, vol. 1, pp. 541546, March 2004.

[16] J. L. Deng, "Introduction to Grey system theory," Journal of Grey System, vol. 1, pp. 1-24, 1989.

[17] J. D. Morales, U. Pineda-Rico, and E. Stevens-Navarro, "Performance comparison between MADM algorithms for vertical handoff in 4G networks," in Proceedings of the 7th International Conference on Electrical Engineering, Computing Science and Automatic Control (CCE '10), pp. 309-314, September 2010.

[18] T. Oliveira, S. Mahadevan, and D. P. Agrawal, "Handling network uncertainty in heterogeneous wireless networks," in Proceedings of IEEE International Conference on Computer Communications (INFOCOM '11), pp. 2390-2398, April 2011.

[19] J. Lilly, "Takagi-sugeno fuzzy systems," in Fuzzy Control and Identification, pp. 88-105, Wiley-IEEE Press, 2010.

[20] E. H. Mamdani, "Application of fuzzy algorithms for control of simple dynamic plant," Proceedings of the Institution of Electrical Engineers, vol. 121, no. 12, pp. 1585-1588, 1974.

[21] J. Zander and S. Kim, Radio Resource Management in Wireless Networks, Artech House, 2001. 

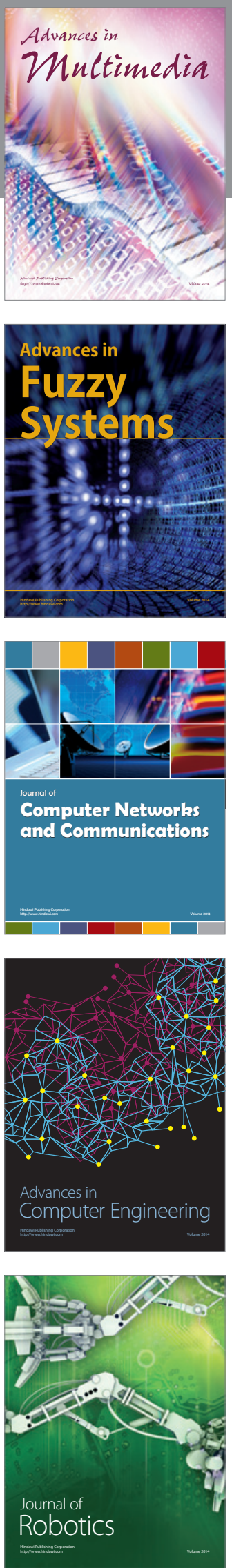

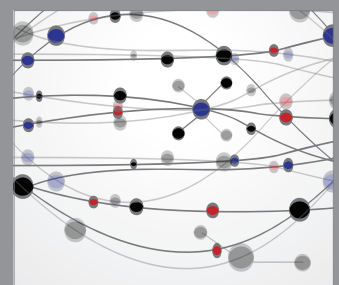

The Scientific World Journal
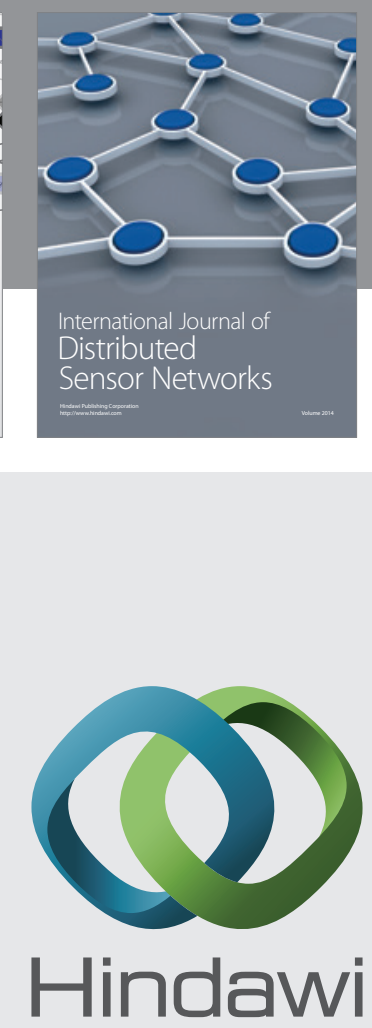

Submit your manuscripts at

http://www.hindawi.com
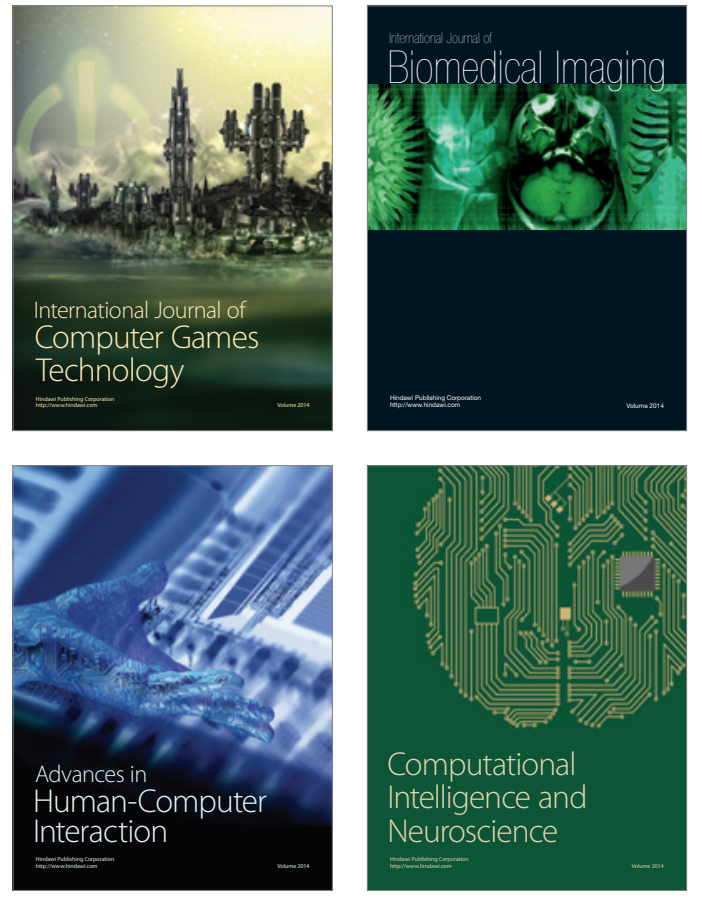
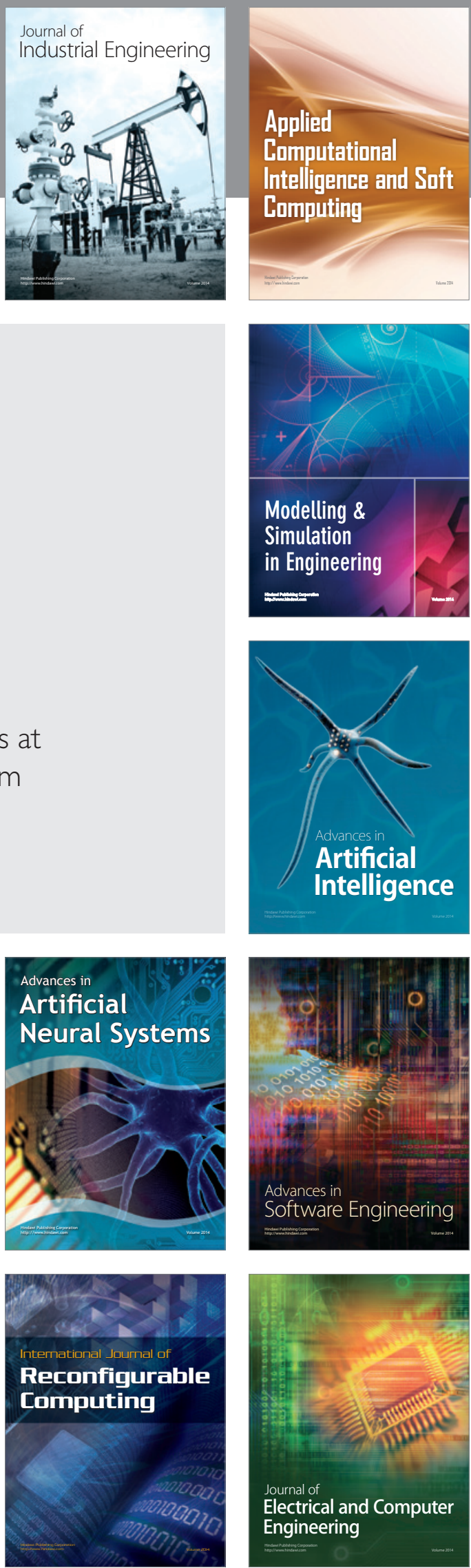